\title{
On the Dependence of Stark Width and Shift on the Ionization Potential
}

\author{
M. S. Dimitrijević and N. Konjević \\ Institute of Applied Physics, Beograd, Yugoslavia \\ Z. Naturforsch. 39a, 553-555 (1984); received January 20, 1984
}

\begin{abstract}
The simple relations between the Stark broadening parameters and the ionization potential derived by Puric et al. [1] are discussed, and attention is drawn to the serious limitations for their application. The periodic dependence of the Stark width and shift on nuclear charge number obtained by the same authors [2] is also explained.
\end{abstract}

Lakićević and Purić (Ref. [3] and references therein) have found that the Stark width $\omega$ and the shift $\delta$ (in units of angular frequency) of spectral lines emitted by a non-hydrogenic atom (or ion) depend on the "effective ionization energy" $I \mid \mathrm{eV}$ $=I_{0}-E_{\mathrm{f}}\left(I_{0}\right.$ and $E_{\mathrm{f}}$ denote the ionization energy and the energy of the lower level, respectively, both counted from the ground level) in the general form of a power series:

$$
\omega=\sum_{k} A_{k} I^{-k} \quad \text { and } \quad \delta=\sum_{k} B_{k} I^{-k} .
$$

On the basis of an analysis of various experimental (in the temperature range $9500-34000 \mathrm{~K}$ ) and theoretical data at $20000 \mathrm{~K}$, Purić et al. [1] found that, for the resonance lines of singly charged ions, the relations (1) become

$w \approx 2050 I^{-4} \AA \quad$ and $\quad d \approx 2050 I^{-4} \AA \mid(2 \mathrm{a}, \mathrm{b})$

These equations (and the corresponding ones for neutral atoms) differ from $(1 \mathrm{a}, \mathrm{b})$ in two respects:

(i) They refer to the values of $w$ and $d$ in units of wavelength and

(ii) they are the result of an average over the elements.

Moreover, $(2 \mathrm{a}, \mathrm{b})$ apply to the particular plasma conditions $N_{\mathrm{e}}=10^{17} \mathrm{~cm}^{-3}$ and $T=20000 \mathrm{~K}$.

Recently, Purić et al. [2] have demonstrated that in the special case of the resonance lines (where $E_{\mathrm{f}}=0$ and therefore $I=I_{0}$ ) of singly charged ions, $w$ and $d$ (in units of the wavelength) depend on the nuclear charge number $Z$ in a manner corresponding to the periodicity of $I_{0}$ in the periodic table of the

Reprint requests to Dr. Milan Dimitrijević, Institute of Physics, P.O. Box 57, 11001 Beograd, Jugoslawien. elements. In order to obtain this dependence, Purić et al. [2] supplemented the available experimental and theoretical values of $w$ and $d$ by interpolated ones. The basis of this interpolation is, for example, in the case of the resonance lines of singly charged ions, $(2 \mathrm{a}, \mathrm{b})$ of the present paper.

We discuss here the following questions: (i) Why is the parameter $I_{0}-E_{\mathrm{f}}$ and not e.g. $I_{0}-E_{\mathrm{i}}$ or $I_{0}$ the best parameter for the description of the $w$ and $d$ dependence on the ionization energy? (ii) Do the Stark broadening parameters, if presented in the wavelength scale, depend on the ionization energy in a simple way, as it is asserted especially for the resonance lines in [1]? (iii) Is the finding in [2] correct that the Stark broadening parameters of the resonance lines depend on the nuclear charge number in a manner corresponding to the periodicity of the ionization energy in the periodic table of the elements?

Equations $(1 \mathrm{a}, \mathrm{b})$ are based on three different approaches (semiempirical, semiclassical and adiabatic) and are understood to hold for isolated neutral atom lines as well as for isolated ion lines. In order to simplify the discussion we shall use the semiempirical formula [4] for the widths and shifts of isolated ion lines in a form convenient for the low temperature limit. In this case the Stark line width (HWHM) in angular frequency units is given by

$$
\begin{aligned}
& \omega \approx 4\left(\frac{\pi}{3}\right)^{3 / 2} \frac{\hbar a_{0}}{m} N\left(\frac{E_{\mathrm{H}}}{k T}\right)^{1 / 2} \\
& \cdot\left[\left\langle i\left(\frac{r}{a_{0}}\right)^{2} \mid i\right\rangle \bar{g}_{\mathrm{th}}+\left\langle f\left(\frac{r}{a_{0}}\right)^{2} \mid f\right\rangle \bar{g}_{\mathrm{th}}\right],
\end{aligned}
$$

where

$$
\left\langle j\left(\frac{r}{a_{0}}\right)^{2} j\right\rangle=\frac{n_{j}^{* 2}}{2 z^{2}}\left[5 n_{j}^{* 2}+1-3 l_{j}\left(l_{j}+1\right)\right] ; j=i, f .
$$


$g_{\text {th }}=0.2$ is the threshold value of the Gaunt factor, and $n *$ is the effective principal quantum number.

Starting from (3) we can easily derive the expression

$$
\text { () } \begin{aligned}
\approx \frac{A N}{T^{1 / 2}} & {\left[\frac{\chi}{\left(I_{0}-E_{\mathrm{i}}\right)^{2}}+\frac{\chi}{\left(I_{0}-E_{\mathrm{f}}\right)^{2}}\right.} \\
& \left.+\frac{\beta_{\mathrm{i}}}{\left(I_{0}-E_{\mathrm{i}}\right)}+\frac{\beta_{\mathrm{f}}}{\left(I_{0}-E_{\mathrm{f}}\right)}\right],
\end{aligned}
$$

where

$$
\begin{aligned}
& A=4\left(\frac{\pi}{3}\right)^{3 / 2} \frac{\hbar a_{0}}{m}\left(\frac{E_{\mathrm{H}}}{k}\right)^{1 / 2} 0.2, \\
& \alpha=\frac{1}{2}\left(5 E_{\mathrm{H}}^{2} z^{2}\right), \\
& \beta_{j}=\left[1-3 l_{j}\left(l_{j}+1\right)\right] E_{\mathrm{H}} / 2 ; \quad j=i, \mathrm{f} .
\end{aligned}
$$

By expanding the denominators on the right-hand side of (4) into geometric series in $I$ [cf. 3, 6],

$$
I^{\prime}=I_{0}-E_{\mathrm{i}}
$$

or $I_{0}$, it is easily possible to make this expression look like a power series in these variables:

$$
\begin{aligned}
\omega & \approx \sum_{k} A_{k}\left(E_{\mathrm{i}}, E_{\mathrm{f}} ; z ; l_{\mathrm{i}}, l_{\mathrm{f}}\right) I^{-k} \\
& \left.=\sum_{k} A_{k}^{\prime}\left(E_{\mathrm{i}}, E_{\mathrm{f}} ; z ; l_{\mathrm{i}}, l_{\mathrm{f}}\right) I^{\prime}\right)^{-k} \\
& =\sum_{k} A_{0 k}\left(E_{\mathrm{i}}, E_{\mathrm{f}} ; z ; l_{\mathrm{i}}, l_{\mathrm{f}}\right) I_{0}^{-k} .
\end{aligned}
$$

However, the arguments (other than $l, z$ ) of the coefficients and with them the coefficients themselves depend - in general - on $I, I^{\prime}$ or $I_{0}$, respectively. Therefore, in the framework of the present approximation ( 1 a) would follow only, if the coefficients $A_{k}\left(E_{\mathrm{i}}, E_{\mathrm{f}} ; z ; l_{\mathrm{i}}, l_{\mathrm{f}}\right)$ would turn out to be constant. Even within groups of lines corresponding to the same transitions in homologous atoms, the latter condition is not fulfilled. The averaging of the coefficients $A_{k}$ over the lines of such a group would lead, of course, to constant coefficients $\bar{A}_{k}$, but this process is apt to smooth out trends and variations.

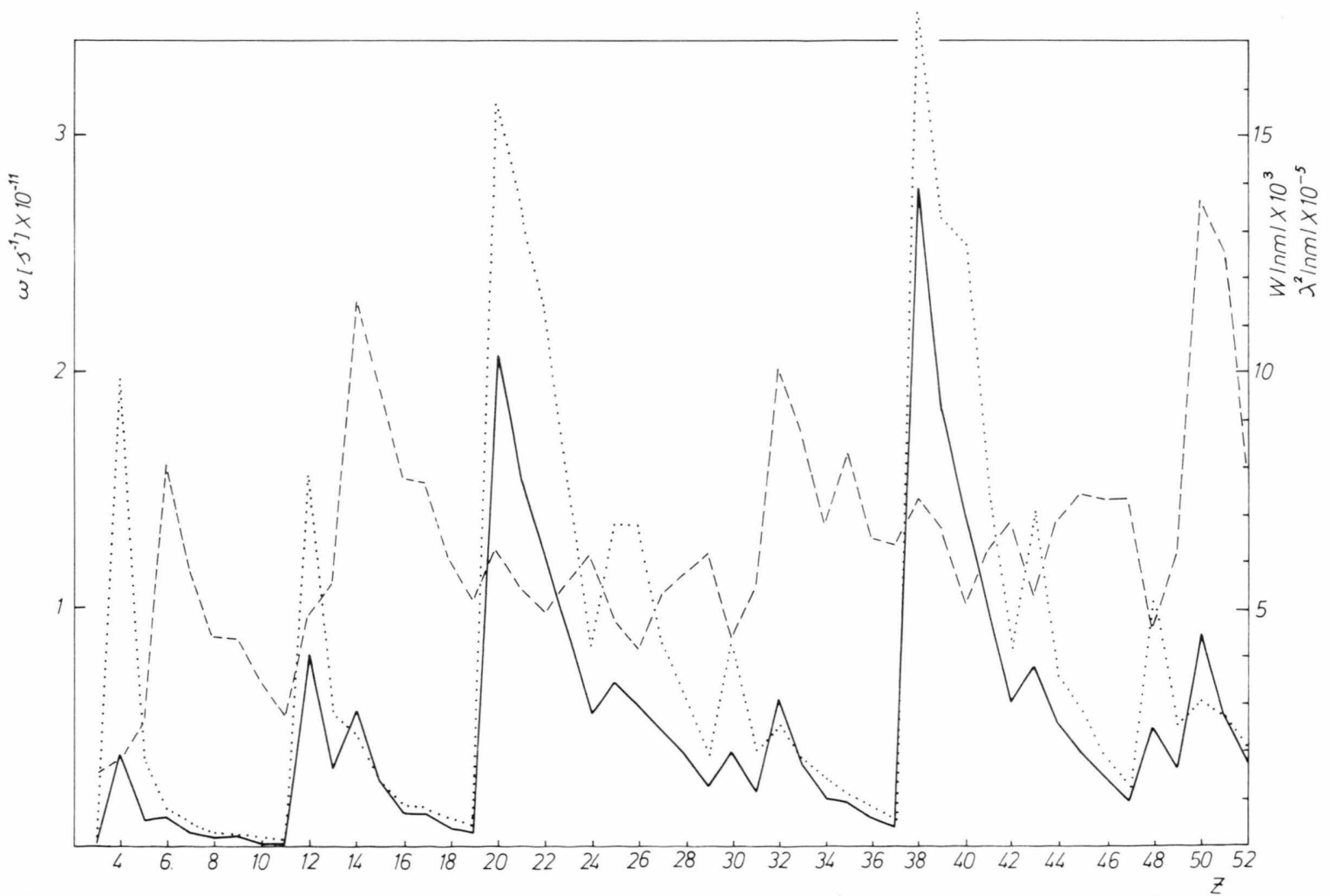

Fig. 1. Stark half-halfwidths ( $w$ in nanometers (solid line), $\omega$ in seconds ${ }^{-1}$ (broken line)) versus nuclear charge number $Z$ for the resonance lines of singly charged ions. The Stark widths are calculated from (2a) and refer to $N_{\mathrm{e}}=10^{17} \mathrm{~cm}^{-3}$ and $T=20000 \mathrm{~K}$. The dependence of $i^{2}$ on $Z$ (dotted line) is also shown. 
When the Stark width is expressed in the wavelength scale:

$$
w(\AA)=i^{2} \frac{\omega\left(\mathrm{s}^{-1}\right)}{2 \pi c} 10^{-8},
$$

as is done in (2a), an additional effect, namely the implicit dependence of the wavelength $i$ on the ionization energy $I_{0}$ becomes important: With the increase of $I_{0}$ the distance between energy levels increases as well, and i becomes smaller. This effect is not taken into account in (2a) and in [7]. Therefore, $(2 \mathrm{a}, \mathrm{b})$ and the corresponding results in [7] should be met with great caution, because they are lacking any theoretical basis.

Recently, Puric et al. [2] demonstrated also the periodic dependence of the Stark widths and shifts in a wavelength scale on the nuclear charge number for neutral and ion resonances. In order to explain their results and to demonstrate the dependence of $i$ on $I_{0}$, the dependence of the Stark widths for the resonance lines of singly ionized atoms upon the nuclear charge number $Z$ is plotted in Figure 1. The Stark broadening data in this figure are evaluated from (2a) and are expressed in nanometers as in [2].

[1] J. Purić, I. Lakićević, and V. Glavonjić, Phys. Lett. $76 \mathrm{~A}, 128$ (1980).

[2] J. Purić and I. S. Lakićević, Phys. Lett. 91 A, 345 (1982).

[3] I. S. Lakićević and J. Purić, in "Spectral Line Shapes II", p. 147, ed. K. Burnett, W. de Gruyter, Berlin 1983.

[4] H. R. Griem, Phys. Rev. 165, 258 (1968).

[5] D. R. Bates and A. Damgaard, Phil. Trans. Roy. Soc. London (Ser. A) 242, 101 (1949).
The same data are also plotted in angular frequency units together with the dependence of $i^{2}$ upon $Z$ for the same resonance lines.

It is clear from Fig. 1 that the dependence upon the nuclear charge number is completely different when Stark broadening parameters are given in angular frequency units. The dependence on $Z$ obtained in [2] for $w$ (and $d$ ) in nanometers is in fact the periodic dependence of $i^{2}$ on $Z$, and it can be easily explained by means of (5) where, for the calculation of the line width, one uses the product of the two quantities $i^{2}$ and $\omega\left(\mathrm{s}^{-1}\right)$. The latter changes much less from element to element. Since the energy difference between the upper and the ground state for the resonance transition (which is used in calculating i) depends implicitly on the ionization energy, the results obtained (Figs. 1 and 2 in [2]), are only an indirect illustration of the well known periodic dependence of the ionization potential upon the nuclear charge number (see e.g. [8]).

\section{Acknowledgement}

The authors gratefully acknowledge valuable comments and suggestions of the referee.

[6] I. S. Lakićević, in "The Physics of Ionized Gases", p. 483, ed. G. Pichler, Institute of Physics of the University, Zagreb 1982.

[7] J. Purić, O. Labat, and I. Lakićević, in Spectral Line Shapes, p. 249, ed. B. Wende, Walter de Gruyter, Berlin 1981.

[8] G. Herzberg, Atomic Spectra and Atomic Structure Dover Publications, New York 1944. 